\title{
Improved mood and sustained attention following acute consumption of Concord grape juice in young, healthy adults: a randomised, placebo-controlled, double-blind, cross-over study
}

\author{
C. F. Haskell and R. Stuart \\ Brain, Performance and Nutrition Centre, Northumbria University, Newcastle upon Tyne, NE1 8ST, UK
}

Berry-derived polyphenols found in purple grape juice have been associated with a number of health benefits in humans, including better episodic memory ${ }^{(1)}$ and improved endothelial function ${ }^{(s e e} 2$ for review). Previous intervention studies of Concord grape juice have demonstrated improvement to memory in age-associated mild cognitive impairment following at least 12 weeks supplementation, as well as increased brain activation (assessed with fMRI) following 16 weeks intervention. Anthocyanin-rich berry extracts have also been observed to improve sustained attention when measured acutely in healthy young adults ${ }^{(3)}$ but no studies to date have demonstrated acute cognitive effects of grape juice.

This randomised, placebo-controlled, double-blind, balanced-crossover study, assessed the effects of a single dose of $200 \mathrm{ml}$ Concord purple grape juice or sugar and flavour-matched placebo in 20 healthy young adults. Computerised measures of cognition and mood were completed at baseline and following a 20-min absorption period, chosen due to a peak in native anthocyanins at $\sim 30$ minutes post-ingestion.
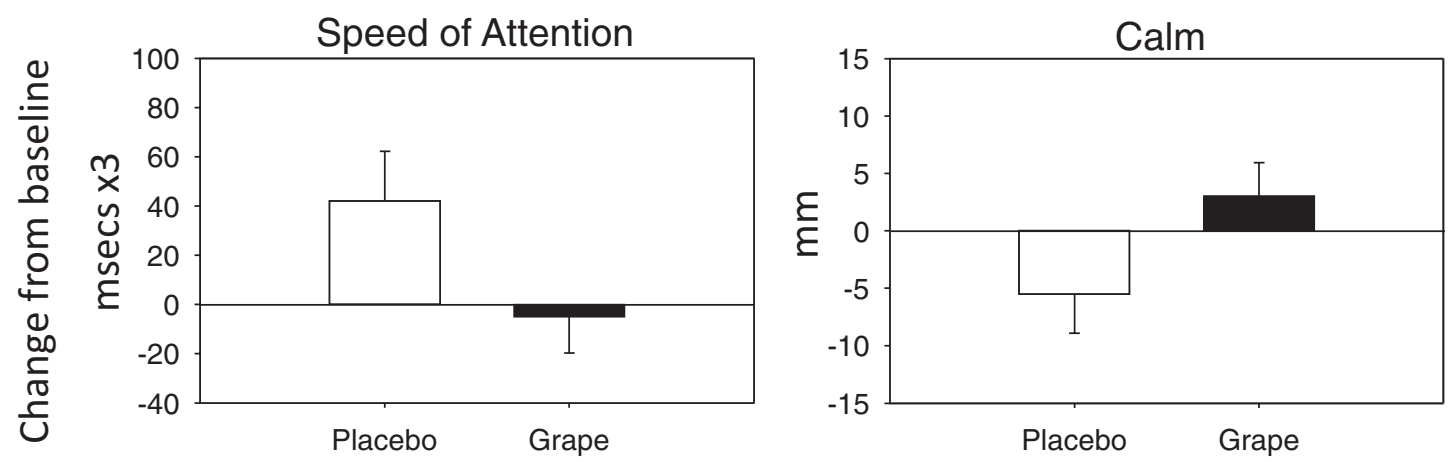

Following a single serve of Concord purple grape juice, a significant increase in calm ratings $(p<0.05)$ and an improvement in speed of attention $(p<0.05)$ were observed. There were no effects on memory. This supports a previous demonstration of improved sustained attention following berry fruit, possibly implicating this as an anthocyanin effect, which account for $46 \%$ of the polyphenolic content of the grape juice administered. However, the phenolic acids, flavanols and flavonols also present are liable to play a role in any neurocognitive effect. These findings in a small sample of healthy young adults suggest that further investigation of the efficacy of purple grape juice in preventing age-associated cognitive decline is warranted to ascertain peak dose effects as well as exploring the active compound(s) responsible for such effects.

1. Kesse-Guyot E, Fezeu L, Andreeva VA, Touvier M, Scalbert A, Hercberg S et al. (2012) Total and Specific Polyphenol Intakes in Midlife Are Associated with Cognitive Function Measured 13 Years Later. Journal of Nutrition 142, 76-83.

2. Vislocky LM \& Fernandez ML (2010) Biomedical effects of grape products. Nutrition Reviews 68, 656-70.

3. Watson AWKD, Haskell CF, Scheepens A (2012) A double blind placebo controlled study measuring the effect of two berry fruit extracts on mood, cognition and monoamine oxidase B inhibition in healthy young adults. Appetite 59, 636. 